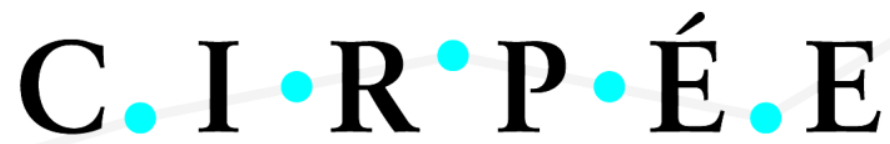

Centre Interuniversitaire sur le Risque, les Politiques Économiques et l'Emploi

Cahier de recherche/Working Paper 12-22

\section{Expected Poverty Changes with Economic Growth and Redistribution}

\author{
Abdelkrim Araar
}

Avril/April 2012

Araar : Département d'économique and CIRPÉE, Université Laval, Québec, Canada aabd@ecn.ulaval.ca

Financial support was provided by the Poverty and Economic Policy (PEP) Research Network, which is financed by the Government of Canada through the International Development Research Centre and the Canadian International Development Agency, and by the Australian Agency for International Development. 


\begin{abstract}
:
This paper focusses on the theoretical and computational framework in order to estimate the impact of economic growth or that of the change in inequality on poverty. During the last few years, there was a growing interest to perform such estimations and to anticipate the implication of some strategic policies, that can be adopted to meet the Millennium Development Goal (MDG, henceforth), that is to cut poverty by half. As is illustrated in this paper, estimated poverty changes may be less precise or even wrong. Precisely, this bad estimation occurs when the distributive changes are non-marginal, whereas the used approach is based on the assumption of marginal changes. In an other case, and where the estimation is implicitly based on a parameterized model of the income distribution, results may be less precise when the predicted distribution cannot reproduce perfectly that derived with the sample. In this study, by using some popular methods, we have used some household surveys of the African countries, as well as, fictive data to show the error size that can occur. Further, we propose a new numerical method to allow to estimate accurately the impact of distributive changes on poverty.
\end{abstract}

Keywords: Poverty, inequality, poverty elasticities, redistribution

JEL Classification: D63, I32, O12 


\section{Introduction}

Algebraically, one can use the tangent or the first derivative of a given function to assess the impact of marginal change of the independent variable on the dependant variable. Kakwani (1993) has developed the theoretical framework to assess the impact of a marginal change in average income -growth- or in inequality -redistribution- on poverty. This work was followed by others to show the impact of other patterns of change on poverty 1 . The main assumption in these works was the marginal nature of the simulated distributive change. However, empirical works do not give much attention to this assumption and the developed methods were applied even if the simulated changes are not marginal.

The literature looking at the nexus between growth, redistribution and poverty distinguishes between two forms of explorations. The first one, is retrospective and aims to show the contribution of growth and redistribution to the observed change in poverty between two periods 2 . The second form is prospective and concerns the projection of poverty by using the most updated distribution of income and by simulating a predefined scheme of change in the distribution of incomes.

There are two main estimates of interest for the prospective form. The first concerns simply the estimation of change in poverty implied by the change in the income distribution. This impact is also called the semi-elasticity of poverty with respect to a given component of the distributive change, like growth. The other estimate of interest is the elasticity of poverty with respect to a given component. While the first estimate focusses on the absolute level of change in poverty, the second shows the relative change according to the initial level of poverty. Klasen and Misselhorn (2008) discussed the advantage of estimating the semielasticity. Indeed, the semi-elasticity is a straightforward indicator to anticipate poverty reduction across regions and on the global level, and this is critical for assessing the progress towards meeting the first Millennium Development Goal. Usually, the MDG targets higher -non marginal- reductions in poverty, which requires larger increases in long-term economic growth. This justifies also the importance to look for an accurate method to estimate the expected poverty changes with the simulated non marginal distributive changes.

In this paper, our main objective is to recall the different methods used to assess the projected change in poverty and their performance. Obviously, even if the performance of these methods is discussed with the semi-elasticity estimator,

\footnotetext{
${ }^{1}$ See for this, Essama-Nssah and Lambert (forthcoming), Son (2006) and Araar and Duclos (2010)

${ }^{2}$ See for instance Datt and Ravallion (1992), Duclos and Araar (2006) and Shorrocks (1999).
} 
these results are sufficient to develop a clear idea about the performance of these methods for the estimation of elasticity 3 .

The rest of the paper is organized as follow. In the next section, we will present the theoretical framework for assessing the expected poverty changes with growth or redistribution. In this section, we propose also a new numerical approach, which is based mainly on the estimation of the density of distribution of incomes with the corrected boundary Gaussian Kernel estimator. In Section 3, we will illustrate the different methods by using real household surveys and fictive data. Finally, some concluding remarks are reported in Section 4.

\section{Anticipated changes in poverty: the theoretical framework}

Formally, for the class of additive poverty indices, if we denote the poverty index by $P(z)$, the change in poverty is defined as follows:

$$
\Delta P(z)=\int_{0}^{z} \pi(z, y) \Delta f(y) d y
$$

where $\pi(z, y)$ denotes the contribution to the total poverty of the individuals with income equals to $y$. For instance, for the FGT index, this contribution is equal to $(1-y / z)_{+}^{\alpha}$ and $x_{+}=\max (x, 0)$. Let $M\left(\gamma_{s}\right)$ be the map of change in incomes with the scheme $s$-growth or redistribution-. We assume that the parameter $\gamma_{s}$ expresses the intensity of changet The semi-elasticity of poverty with respect to $s$ will be defined as follows:

$$
\kappa_{s}=\frac{\partial P}{\partial \gamma_{s}}
$$

whereas the elasticity of poverty with respect to $s$ is given by:

$$
\epsilon_{s}=\frac{\kappa_{\gamma_{s}}}{P}
$$

The total impact on poverty implied by distributive change with the map $M\left(\gamma_{s}\right)$ is:

$$
\Delta P(z)=\kappa_{\gamma_{s}} d \gamma_{s}
$$

\footnotetext{
${ }^{3}$ Note that the elasticity is simply the estimated semi-elasticity normalized by the initial level of poverty.

${ }^{4}$ For instance, in the case of growth, we have that $M\left(\gamma_{\text {growth }}\right): y_{i} \rightarrow y_{i}\left(1+\gamma_{\text {growth }}\right)$.
} 


\subsection{The counterfactual approach}

At this stage, we present the estimation methods by beginning with the counterfactual approach. This approach is based on the estimation of the difference between the poverty under the counterfactual distribution and that of the initial distribution. For instance, if our aim is to estimate the change in poverty generated by $1 \%$ of economic growth, we first begin by constructing the counterfactual distribution. Formally, if we denote the level of economic growth by $g$, the counterfactual income $y^{c}$ can be defined as follows:

$$
y_{g}^{c}=(1+g) y
$$

What is the pattern of change in the distribution in order to simulate the increase in inequality? If inequality is measured by the Gini index, an increase in inequality by $1 \%$, say, can be done in a very large number of ways, each of them involving different transformations of the original income distribution.

The different maps of change in inequality will generate different impacts on poverty, depending on the precise nature of the distributive change. A general type of distributive change that can be handled nicely from an analytical perspective spreads all incomes away from the mean by a proportional factor $\lambda$. It corresponds, roughly speaking, to an increased bi-polarization of incomes away from an unchanged mean 5 . Such bi-polarization is equivalent to adding $(\lambda-1)(y-\mu)$ to each income. This implies also that the counterfactual distribution simulating the redistribution effect is:

$$
y_{r}^{c}=y+\underbrace{(\lambda-1)(y-\mu)}_{\text {increased bipolarization }} .
$$

Note that this bi-polarization does not affect average income. Further, one can easily prove that the proportional increase in Gini index with this scheme is equal to $(\lambda-1) 6$.

Now, if one focusses on the precision of the counterfactual approach, we can expect that, with the availability of large household surveys, the counterfactual approach will work better with both marginal and the non marginal changes. However, there is the exception for the estimation of the impact of change on headcount

\footnotetext{
${ }^{5}$ Here we recall that the derived elasticity of poverty with respect to inequality, proposed by Kakwani (1993) have this scheme of change. For more details, see also Wolfson (1994) and Duclos and Échevin (2005).

${ }^{6}$ See for this Duclos and Araar (2006).
} 
index. Indeed, when the change is marginal, individuals that may escape poverty are those whose income is closest to the poverty line. Formally, their proportion is equal to the level of density function at poverty line. When we use the household surveys, we cannot observe directly this population group since the sample may not contain the observations with incomes exactly equal to the poverty line. In such case, and by assuming the continuity in the distribution of incomes at population level, one can estimate the density function, by using for instance the Kernel estimator, and then, estimate the impact on poverty. In general this method will give more accurate results to be inferred to the whole population, as we can discover later.

\subsection{Marginal changes and analytical approach}

Under the assumption of marginal changes in average income or inequality, the analytical -algebraical- approach may be used to assess the impact of these changes on poverty. These developments are useful to anticipate the impact of different potential governmental reforms on poverty or inequality. For instance, as already indicated, the estimated semi-elasticity of poverty with respect to income growth can serve to assess the impact of an expected economic growth on poverty. Further, this semi-elasticity can also be used to estimate the required growth to achieve a given level of reduction in poverty. When the FGT poverty index is used to assess poverty ${ }^{7}$ and when growth refers to the marginal change in average income, the overall growth semi-elasticity $\left(\kappa_{g}\right)$ of poverty is given by:

$$
\kappa_{g}= \begin{cases}-z f(z) & \text { if } \quad \alpha=0 \\ \alpha[P(z ; \alpha)-P(z ; \alpha-1)] & \text { if } \quad \alpha \geq 1\end{cases}
$$

where $z$ is the poverty line, $f(z)$ is the density function at income level equal to $z$. The overall inequality semi-elasticity $\left(\kappa_{r}\right)$ of poverty when growth is nil is given by:

$$
\kappa_{r}=\left\{\begin{array}{lr}
(\mu-z) f(z) & \text { if } \quad \alpha=0 \\
\alpha[P(z, \alpha)+(\mu / z-1) P(z ; \alpha-1)] & \text { if } \quad \alpha \geq 1
\end{array}\right.
$$

As shown later, this analytical approach gives more accurate results when the simulated growth or redistribution is small.

\footnotetext{
${ }^{7}$ See Foster, Greer, and Thorbecke (1984).
} 


\subsection{The parameterized approach}

Another way to estimate the expected changes in poverty is by modeling the distribution of income. Subsequently, one can derive the intrinsic formulas to define the expected change in poverty resulted from growth or redistribution. The literature proposes different functional forms to model the distribution of incomes. 8 . Among them, the popular Log-Normal distribution function. Bourguignon (2002) shows that when income distribution follows a Log-Normal distribution with an average $\mu$ and a standard deviation $\sigma$, the headcount can be defined as:

$$
P(z ; \alpha=0)=\Phi\left[\left(\frac{\log (z / \mu)}{\sigma}\right)+\frac{\sigma}{2}\right]
$$

where $\Phi$ denotes the cumulative normal distribution function. For the poverty headcount index, its growth semi-elasticity is defined as follows:

$$
\kappa_{g}=\frac{1}{\sigma} \phi\left[\left(\frac{\log (z / \mu)}{\sigma}\right)+\frac{\sigma}{2}\right]
$$

where $\phi($.$) denotes the normal density function. For the poverty gap and using the$ formulas $P(z ; \alpha=1)=\left(1-\mu_{p} / z\right) P(z ; \alpha=0)$, where $\mu_{p}$ denotes the average income within the poor group, we find that:

$$
\kappa_{g}=-\frac{\mu_{p}}{z} \Phi\left[\left(\frac{\log (z / \mu)}{\sigma}\right)+\frac{\sigma}{2}\right]
$$

As showed by Aitchison and Brown (1957), for the Log-Normal distribution, the Gini index is defined as follows:

$$
G=2 \Phi(\sqrt{\sigma / 2})-1
$$

When it is assumed that the change in redistribution does not alter the Log-Normal form of the distribution (changing simply the level of parameter $\sigma$ ), the semielasticity due to inequality changes is defined as follows:

$$
\kappa_{\sigma}=\phi\left[\left(\frac{\log (z / \mu)}{\sigma}\right)+\frac{\sigma}{2}\right]\left[\frac{1}{2}-\left(\frac{\log (z / \mu)}{\sigma^{2}}\right)\right]
$$

Of course, one can use the derivation in chain and assess the semi-elasticity of poverty with respect to the Gini index when the change in inequality is controlled by the parameter $\sigma$ ?

\footnotetext{
${ }^{8}$ See for instance Chotikapanich (2008).

${ }^{9}$ Here we have $\sigma=\sqrt{2} \Phi^{-1}((G+1) / 2)$.
} 
Is the parameterized approach better than the analytical one? As we will discover later in our application, even if the predicted distribution is slightly different from the observed one, the estimated impact of distributive changes on poverty may contain a non neglected error term.

\subsection{The numerical approach}

The numerical approach, proposed in this paper, is based mainly on the estimation of the proxy of the true density function of income distribution. Precisely, for this end, we propose the use of the Gaussian Kernel estimator. Note that the usual Kernel estimator is a straightforward method for estimating the density function without specifying beforehand its form 10 . For more precision in the estimation of this density, we propose to correct the bias of bounded distribution 11 Formally, the expected change in headcount, resulted from economic growth, is equal to:

$$
\Delta P(z ; \alpha=0)=-\int_{z /(g+1)}^{z} f(y) d y
$$

For the numerical computation, one can estimate the kernel density function within the income range $[0, z \sqrt[12]{12}$, then can use the trapezoidal rule for the numerical integration.

How about the impact of growth on poverty gap? This impact may be defined as follows:

$$
\Delta P(z ; \alpha=1)=\underbrace{-\int_{0}^{\frac{z}{1+g}}(g y / z) f(y) d y}_{\mathrm{C} 1}-\underbrace{\int_{\frac{z}{1+g}}^{z}(1-y / z) f(y) d y}_{\mathrm{C} 2}
$$

The component $C 1$ indicates the reduction in poverty gap attributed to the improvement in wellbeing of those that continue to be poor. The component $C 2$ indicates the reduction in poverty gap attributed to those that escape from poverty after the economic growth. When the growth $g$ converges to zero, the component $C 2$ may be neglected. However, neglecting this part when growth is non marginal may induce a non neglected error. Here also, we can use the numerical integration to estimate the two components $C 1$ and $C 2$, and then, we can sum them in order

\footnotetext{
${ }^{10}$ See for instance Silverman (1986) and Duclos and Araar (2006).

${ }^{11}$ For more details, see the Appendix 1 .

${ }^{12}$ For instance, $y \in\{0, a, 2 a, \ldots, n a=z\}$ and $n=1000$.
} 
to assess the total impact of growth on poverty. Using the same approach, one can show that the impact of growth on poverty severity is as follows:

$$
\Delta P(z ; \alpha=2)=\underbrace{-\int_{0}^{\frac{z}{1+g}}\left(g y(g y-2(z-y)) / z^{2}\right) f(y) d y}_{\mathrm{C} 1}-\underbrace{\int_{\frac{z}{1+g}}^{z}(1-y / z)^{2} f(y) d y}_{\mathrm{C} 2}
$$

For the increase in inequality with the bi-polarization scheme and when $z<\mu$, the impact on headcount is:

$$
\Delta P(z ; \alpha=0)=\int_{z}^{(z+(\lambda-1) \mu) / \lambda} f(y) d y .
$$

Thus, in this case, the headcount will increase. When $z>\mu$, we will observe a decrease in headcount and the impact is given by:

$$
\Delta P(z ; \alpha=0)=\int_{(z+(\lambda-1) \mu) / \lambda}^{z} f(y) d y .
$$

As discussed also by Araar and Duclos (2010), the sign of the impact will depend on the difference between the poverty line $(z)$ and the average income $(\mu)$. For the poverty gap and when $z<\mu$, the impact on poverty will take the following form:

$$
\begin{aligned}
\Delta P(z ; \alpha=1)= & \underbrace{\int_{0}^{z}(\lambda-1)((\mu-y) / z) f(y) d y}_{\mathrm{C} 1} \\
& +\underbrace{\left.\int_{z}^{(z+(\lambda-1) \mu) / \lambda}[((z-\mu)+\lambda(\mu-y)) / z)\right] f(y) d y}_{\mathrm{C} 2}
\end{aligned}
$$

In this case, all individuals of the poor group will experience an increase in their poverty depth (component $C 1$ ). In addition, another part of the non poor group will join the poor group (component $C 2$ ). The absolute depth of an individual within this group is equal to $(\lambda-1)(\mu-y)$ corrected by her/his initial surplus $(-(y-z))$. In the case where $z>\mu$, the impact on poverty will take the following form: 


$$
\begin{aligned}
\Delta P(z ; \alpha=1)= & \underbrace{\int_{0}^{\mu}(\lambda-1)((\mu-y) / z) f(y) d y}_{\mathrm{C} 1} \\
& -\underbrace{\left.\int_{\mu}^{(z+(\lambda-1) \mu) / \lambda}[((\lambda-1)(y-\mu)) / z)\right] f(y) d y}_{\mathrm{C} 2} \\
& -\underbrace{\int_{(z+(\lambda-1) \mu) / \lambda}^{z}(1-y / z) f(y) d y}_{\mathrm{C} 3}
\end{aligned}
$$

Here we find three main components. The first concerns the group with income lower than the average and for which the poverty depth increases. The second concerns the group whose income is higher than of the average income, but the improvement in their incomes, due to the distributive change, is not sufficient to enable them to escape from poverty. The third component concerns those that can escape from poverty after the distributive change.

With an increase in inequality based on the bi-polarization scheme and when $z<\mu$, the impact on poverty severity is as follow:

$$
\begin{aligned}
\Delta P(z ; \alpha=2)= & \frac{1}{z^{2}} \underbrace{\int_{0}^{z}\left[(z-(\lambda y-(\lambda-1) \mu))^{2}-(z-y)^{2}\right] f(y) d y}_{\mathrm{C} 1} \\
& +\underbrace{\int_{z}^{(z+(\lambda-1) \mu) / \lambda}\left[(1-y / z)^{2}\right] f(y) d y}_{\mathrm{C} 2}
\end{aligned}
$$

When $z>\mu$, the impact on poverty severity is as follow:

$$
\begin{aligned}
\Delta P(z ; \alpha=2)= & \frac{1}{z^{2}} \underbrace{\int_{0}^{\mu}\left[(z-(\lambda y-(\lambda-1) \mu))^{2}-(z-y)^{2}\right] f(y) d y}_{\mathrm{C} 1} \\
& +\frac{1}{z^{2}} \underbrace{\int_{\mu}^{(z+(\lambda-1) \mu) / \lambda}\left[(z-(\lambda y-(\lambda-1) \mu))^{2}-(z-y)^{2}\right] f(y) d y}_{\mathrm{C} 2} \\
& -\underbrace{\int_{(z+(\lambda-1) \mu) / \lambda}^{z}\left[(1-y / z)^{2}\right] f(y) d y}_{\mathrm{C} 3}
\end{aligned}
$$




\section{Application}

We begin by illustrating the expected impacts of distributive changes on poverty by using two fictive distributions. We denote the first distribution by $A$ and the second by $B$. These distributions were constructed in order to follow the Log-Normal form and each contains 10000 observations. Means of $A$ and $B$ are the same and equal to one. The standard deviation of $A$ is one while that of $B$ is two: this generates more inequality in the second distribution 13 . In Figure 1, we show the link between the poverty gap and the economic growth (proportion of change in average income). In this first application, we use the counterfactual approach. For instance, to estimate the expected poverty gap when economic growth is $20 \%$, we use the initial income distribution multiplied by 1.2. In Figure 2, we show the proportional change in poverty gap according to the economic growth. It is evident that the decrease in poverty is amplified when inequality is low, which is the case for the distribution $A$. As reported above, with marginal changes in

Figure 1: Poverty gap and economic growth

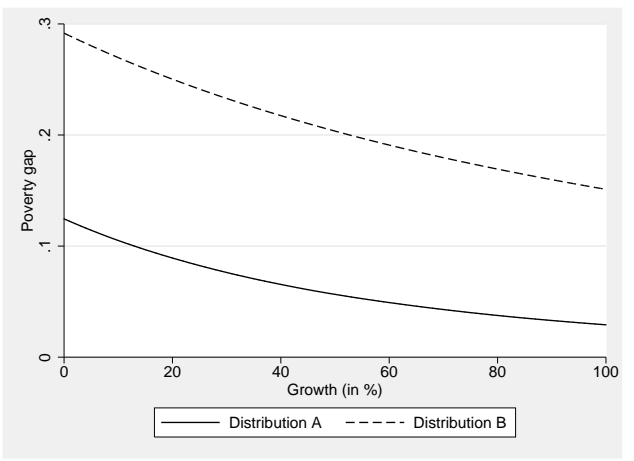

Figure 2: Proportional change in poverty according to growth

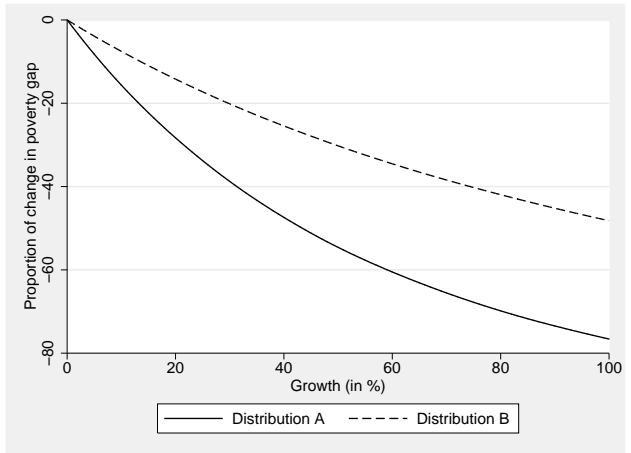

average income, one can use the analytical approach (poverty semi-elasticity with respect to growth) instead of the counterfactual approach, used in Figures 1 and 2 . However, how large is the error size when growth began non marginal? To show this clearly, we present in Figure 3 the estimated proportional change in poverty

\footnotetext{
${ }^{13}$ For more details, see the Appendix 2
} 
Figure 3: The proportional change in poverty with the analytical and counterfactual approaches

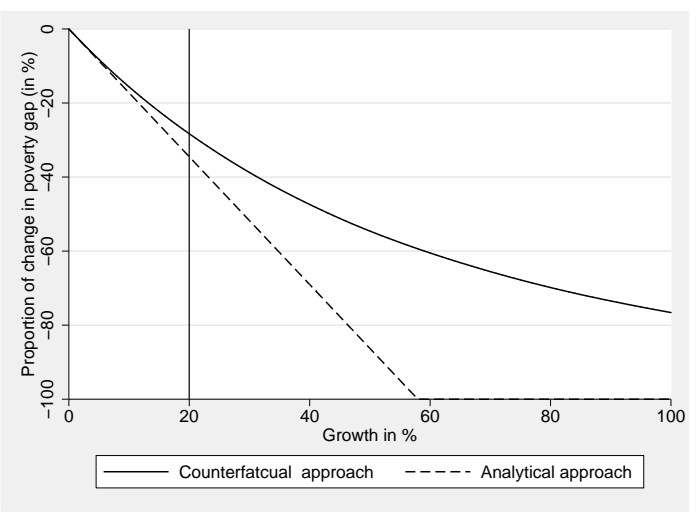

Figure 4: The estimated error in the proportional change in poverty with the analytical approach

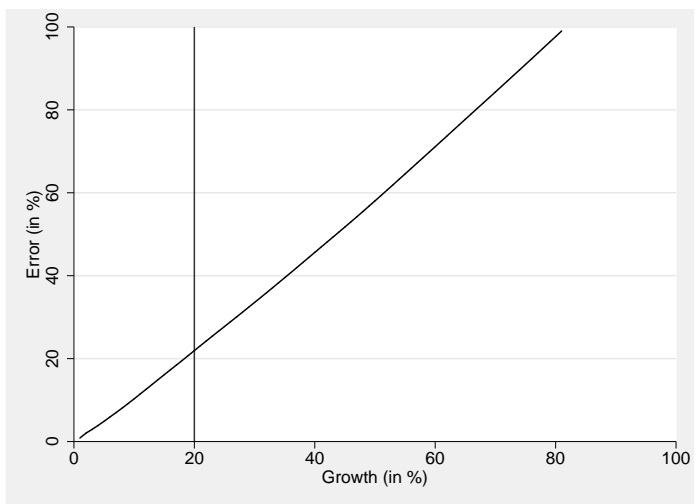

gap with with the counterfactual and the analytical approaches for distribution $A$. Starting from this figure, one can remark that, for small levels of growth, the two approaches will practically give similar results. However, when growth is high (20\% for instance), the estimates diverge. As shown also in Figure 4 with an expected growth of $20 \%$, the error is approximately $20 \%$. In other words, if one uses the Kakwani (1993) approach to estimate the impact of targeted economic growth as by the first millennium development goal, the error of estimation will be high and the counterfactual approach with non marginal changes will give more accurate results. Now, we focus on the change in poverty implied by an expected increase in inequality. As shown in Figures 5 and 6 , the analytical approach tends to underestimate the expected increase in poverty. For instance, when the increase in inequality is $20 \%$ the underestimation is about $7 \%$ with the fictive distribution $A$. At this stage, let us focussing on the implication of using the parameterized models. To this end, we use the national household surveys of Nigeria 2004 and Burkina Faso 1994. For each of these two samples, we assume that per capita expenditures follow the Log Normal distribution. The first exploration consists in checking visually the pertinence of this assumption by plotting the corrected boundary kernel density distribution and the predicted density with the Log Normal model.

As shown in Figure 7, the distribution of per capita expenditures in Nigeria of 2004 is close to that of the Log Normal. Thus, one can expect that the estimated 
impact of growth or redistribution on poverty will contain fewer errors with the parameterized approach. However, this conclusion is not true in all cases. For instance, in Figure 8, with the Burkina's 1994 distribution, the predicted distribution with the Log-Normal model is far from the true distribution; this is true in different parts of the distribution.

Figure 5: The proportional change in poverty gap with the analytical and counterfactual approaches

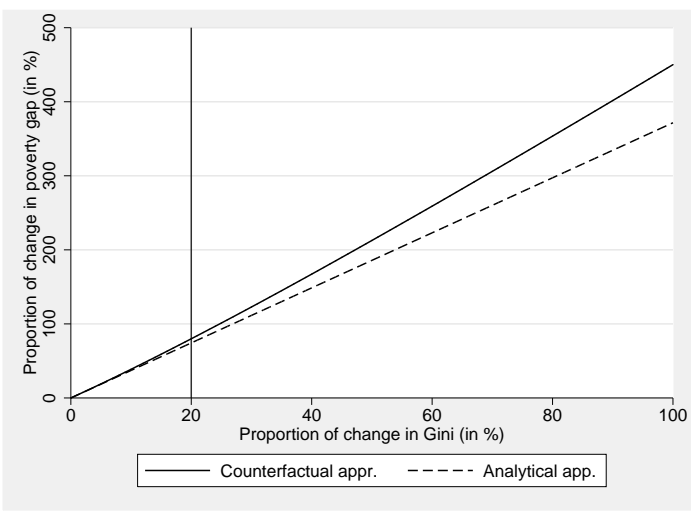

Figure 6: The estimated error in the proportional change in poverty with the analytical approach

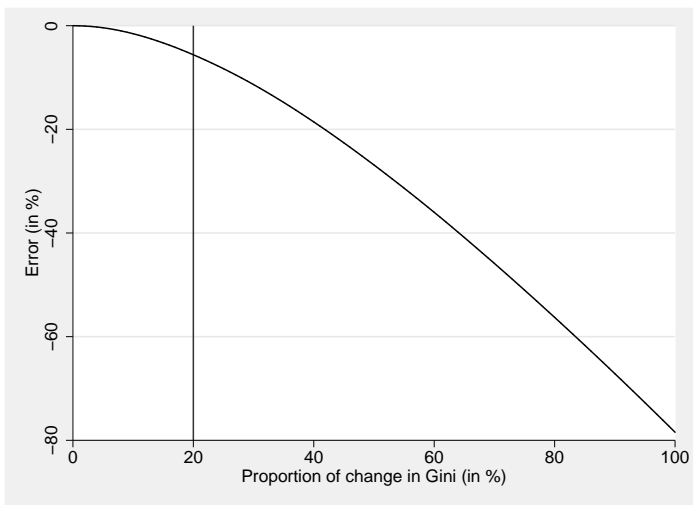

Before estimating the impact of growth or redistribution on poverty, let us focussing simply on the estimation of the FGT index. In addition to the counting approach that uses the discrete data of the sample, one can follow the parameterized approach or the numerical approach. In Figure 9 we estimate the headcount according to the poverty line by using different approaches. The first remark is about the perfect concordance of the true estimates with the numerical approach. As expected, the parameterized model is less flexible and fails to reproduce the true distribution, and thus, the accurate results. With the official poverty line in 1994 (41099 F CFA), when we estimate the headcount with the parameterized approach, the generated error is about $20 \%$. Let us continue with the Burkina Faso survey to estimate the impact of a potential income growth on poverty. To this end, we use the different methods, presented above, in order to show how well each of them performs. These methods are:

A Counterfactual approach: We estimate the change in poverty gap after multiplying the vector income -per capita expenditures- by $(1+g)$, where $g$ 
Figure 7: Estimated density function of per capita expenditures Nigeria (2004)

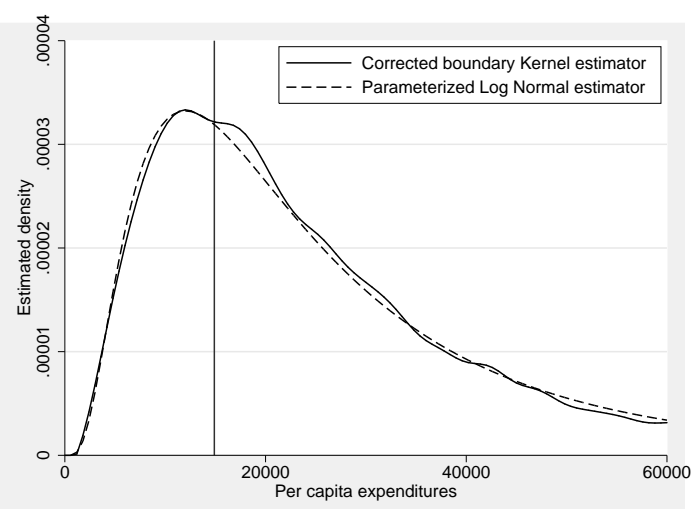

Figure 9: Estimated headcount with the parameterized and discrete approaches: Burkina Faso (1994)

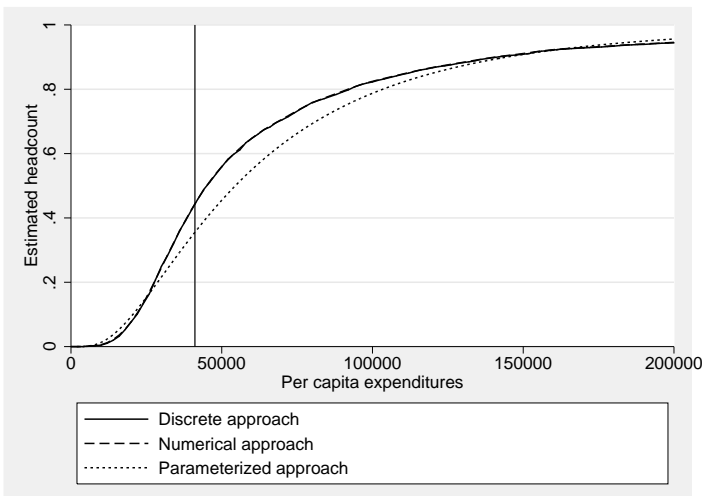

Figure 8: Estimated density function of per capita expenditures Burkina Faso (1994)

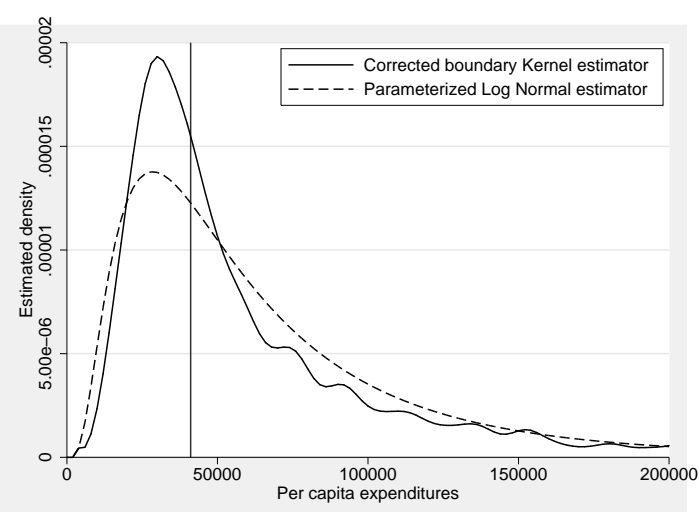

Figure 10: The estimated error in the proportional change in poverty with the parameterized approach

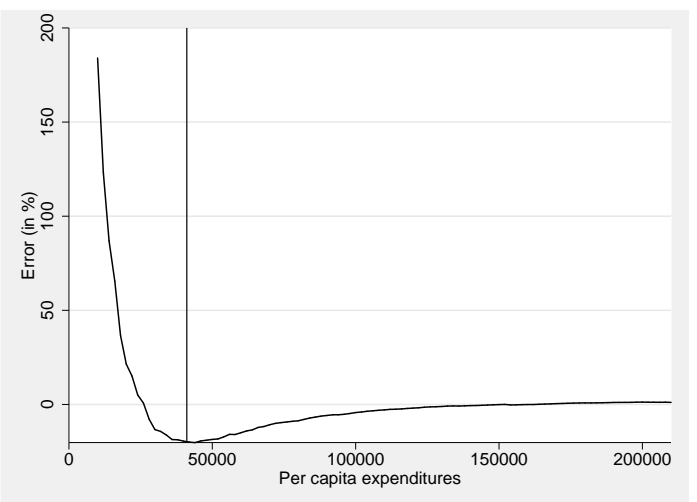


denotes the growth.

B Analytical approach: As indicated in section 2.2, with the analytical approach, the impact of growth on poverty gap equals to $[P(z ; \alpha=1)-$ $P(z ; \alpha=0)] g$. We recall here that, for the growth component, this approach was proposed by Kakwani (1993).

C Parameterized approach: Usually, in empirical applications, this method implies an impact equal to:

$$
\Delta P(z ; \alpha=1)=-\left(\mu_{p} / z\right) P(z ; \alpha=0)
$$

where $\mu_{p}$ denotes the average income of poor group. The headcount $(P(z ; \alpha=0))$ is estimated by following Bourguignon (2002)'s parameterized approach with the assumption of Log-normal distribution of incomes.

D Numerical approach: First, we estimate the third order corrected boundary Gaussian Kernel estimator. Then, we integrate numerically the impact as defined by equation (15).

The results of estimations with the four approaches are reported in Figure 11 Based on this, the main conclusions that one can draw are the following:

- The numerical approach gives more precise results for the marginal and non-marginal distributive changes.

- The analytical approach gives better results when the distributive changes are small. One must be prudent for the application of this approach when the changes are not marginal.

- Based on what was proposed by Bourguignon (2002), we fail in some cases to estimate the accurate changes in poverty because of the limitation to model the income distribution.

At this stage, let us exploring the impact of changes in inequality on poverty. To this end, we continue to use the Burkina's data and we assume that the increase in inequality modeled by the increase in bi-polarization, as defined by equation (6). As shown in Figure 12, while the analytical approach contains a non neglected error with the non-marginal changes, the numerical approach performs well and gives more accurate results.

Note that for the estimation with the different approaches, a set of Stata modules are prepared for users. For more details, see the Appendix 3. 
Figure 11: Estimated impact of growth on poverty gap: Burkina Faso (1994)

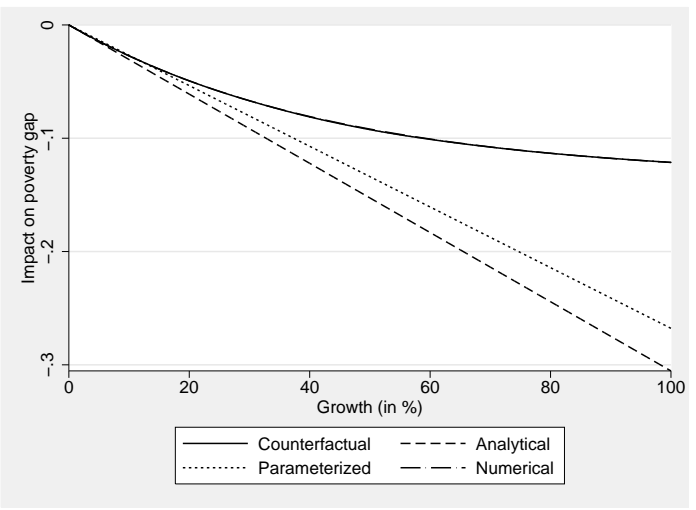

Figure 12: Estimated impact of increase in Gini inequality on poverty gap: Burkina Faso (1994)

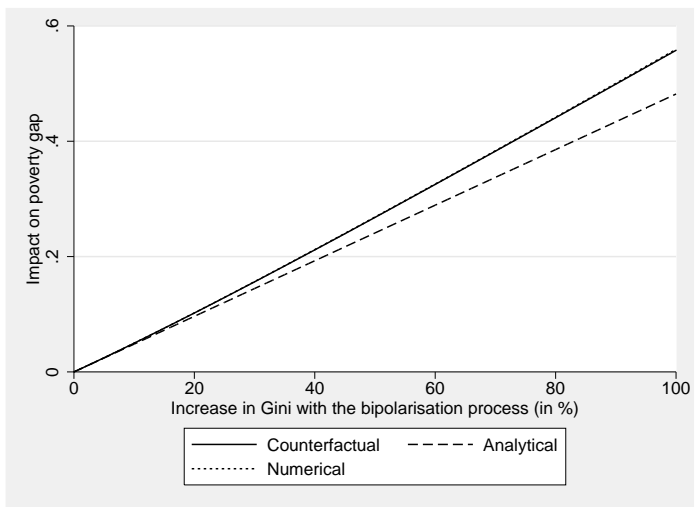

\section{Conclusion and recommendations}

During the last decades, there was an increasing preoccupation within the international community towards the improvement of social wellbeing. Even if the targeted objectives of the MDGs are not likely, for most of the cases, to be reached by 2015 , this international commitment is very benefic to promote the synergy of incitation for stakeholders in general. This synergy constitutes also the main factor inciting national governments to improve the wellbeing of the most deprived groups.

To assess the impact of some potential governmental programs on poverty, we need to use the most updated and accurate methods. In this paper, we recall the methods that were intensively used in empirical works. Obviously, more precise estimations are necessary to have a clear judgment about the social efficiency of different potential reforms and to assess their impact on poverty.

Amongst the objectives of this paper is to show the limitations of some methods used to estimate the impact of distributive changes on poverty, and where these changes result from growth or redistribution -inequality-. Also, we propose a new approach based on the numerical estimation of the impact. In summary, the main conclusions found in this paper are:

- With the non-marginal distributive changes, the use of the analytical approach will induce a non neglected error in our estimates. This can be 
explained by the non linear link between poverty indices and components controlling for the change in distribution, like growth.

- The parameterized approach, proposed by Bourguignon (2002), will in general generates a non neglected error term in the estimated impact. This is especially the case when the predicted distribution is different from the observed one.

- The numerical approach, proposed in this paper, gives accurate results for the two forms of change (marginal and non-marginal). This numerical approach is promising in the sense that it can be extended to study other topics of the distributive analysis. 


\section{References}

Aitchison, J. And J. Brown (1957): The Lognormal Distribution: With Special Reference to Its Uses in Economics, Cambridge University Press.

ARAAR, A. AND J.-Y. DuClos (2010): "Poverty and inequality: a micro framework," Journal of African Economies, ejq005.

Bearse, P., C. J. AND P. Rilstone (2007): "Estimation of Duration Models With Unobserved Heterogeneity Efficient Semi parametric," Econometric Theory, 23, 281-308.

BourguignON, F. (2002): “The growth elasticity of poverty reduction : explaining heterogeneity across countries and time periods," Tech. rep.

Chotikapanich, D., ed. (2008): Modeling Income Distributions and Lorenz Curves Series: Economic Studies in Inequality, Social Exclusion and Well-Being, vol. 5, New York ; London: Springer.

DATT, G. AND M. RAVAllion (1992): “Growth and Redistribution Components of Changes in Poverty Measures: a Decomposition with Applications to Brazil and India in the 1980's," Journal of Development Economics, 38, 275-295.

Duclos, J.-Y. AND A. ARAAR (2006): Poverty and Equity Measurement, Policy, and Estimation with DAD, Berlin and Ottawa: Springer and IDRC.

Duclos, J.-Y. AND D. ÉCHEvin (2005): "Bipolarization Comparisons," Economics Letters, 87, 249-258.

ESSAMA-NsSAH, B. AND P. J. LAMBERT (forthcoming): "Measuring propoorness: a unifying approach with new results," Review of Income and Wealth.

Foster, J., J. Greer, And E. Thorbecke (1984): “A Class of Decomposable Poverty Measures,” Econometrica, 52, 761-776.

JONES, M. C. (1993): "Simply boundary correction for Kernel density estimation," Statistics and Computing, 3, 135-146.

KAKWANI, N. (1993): "Poverty and Economic Growth with Application to Cote d'Ivoire," Review of Income and Wealth, 39, 121-39.

Klasen, S. And M. Misselhorn (2008): "Determinants of the Growth Semi-Elasticity of Poverty Reduction,” Ibero America Institute for Econ. 
Research (IAI) Discussion Papers 176, Ibero-America Institute for Economic Research.

ShORROCKS, A. (1999): "Decomposition procedures for distributional analysis: A unified framework based on the Shapley value," Tech. rep., University of Essex.

Silverman, B. (1986): Density Estimation for Statistics and Data Analysis, London: Chapman and Hall.

Son, H. H. (2006): "Assessing the "Pro-Poorness" of Government Fiscal Policy in Thailand," Public Finance Review, 34, 427-449.

Wolfson, M. (1994): "When Inequalities Diverge," American Economic Review, 84, 353-58. 


\section{Appendix 1 The corrected boundary Gaussian Ker- nel estimator}

The Gaussian kernel estimator of a density function $f(x)$ is defined by:

$$
\hat{f}(x)=\frac{\sum_{i} w_{i} K_{i}(x)}{\sum_{i=1}^{n} w_{i}}
$$

where

$$
K_{i}(x)=\frac{1}{h \sqrt{2 \pi}} \exp \left(-0.5 \lambda_{i}(x)^{2}\right) \quad \text { and } \quad \lambda_{i}(x)=\frac{x-x_{i}}{h}
$$

where $h$ is a bandwidth that acts as a "smoothing" parameter. A problem occurs with kernel estimation when a variable of interest is bounded. It may be for instance that consumption is bounded between two values, a minimum and a maximum, and that we wish to estimate its density "close" to these two bounds. If the true value of the density at these two bounds is positive, usual kernel estimation of the density close to these two bounds will be biased. One way to alleviate these problems is to use a smooth "corrected" Kernel estimator, following a paper by Bearse and Rilstone (2007) (See also Jones (1993)). A boundary-corrected Kernel density estimator can then be written as:

$$
\hat{f}(x)=\frac{\sum_{i} w_{i} K_{i}^{*}(x) K_{i}(x)}{\sum_{i=1}^{n} w_{i}}
$$

The scalar $K_{i}^{*}(x)$ is defined as:

$$
\begin{gathered}
K_{i}^{*}(x)=\psi(x)^{\prime} P\left(\lambda_{i}(x)\right) \\
P(\lambda)=\left(\begin{array}{ccccc}
1 & \lambda & \frac{\lambda^{2}}{2 !} & \cdots & \frac{\lambda^{s-1}}{(s-1) !}
\end{array}\right) \\
\psi(x)=M^{-1} l_{s}^{\prime}=\left(\int_{A}^{B} K(\lambda) P(\lambda) P(\lambda)^{\prime} d \lambda\right)^{-1} l_{s}
\end{gathered}
$$




$$
A=\frac{x-\max }{h}, \quad B=\frac{x-\min }{h}, \quad l_{s}^{\prime}=(1,0,0 \cdots, 0)
$$

min is the minimum bound, and $\max$ is the maximum one. This correction will remove the boundary bias to order $h^{s}$.

\section{Appendix 2 The Log-Normal income distribution}

If $x \sim N\left(\mu_{x}, \sigma_{x}^{2}\right)$, the $y=e^{x} \sim L N\left(\mu_{y}, \sigma_{y}^{2}\right)$, and where:

- $\mu_{y}=e^{\mu_{x}+0.5 \sigma_{x}^{2}}$

- $\sigma_{y}^{2}=e^{2 \mu_{x}+\sigma_{x}^{2}}\left(e^{\sigma_{x}^{2}}-1\right)$

Conversely, $\mu_{x}$ and $\sigma_{x}^{2}$ can be found from $\mu_{y}$ and $\sigma_{y}^{2}$ as follows:

- $\mu_{x}=2 \ln \left(\mu_{y}\right)-0.5 \ln \left(\sigma_{y}^{2}+\mu_{y}^{2}\right)$

- $\sigma_{x}^{2}=-2 \ln \left(\mu_{y}\right)+\ln \left(\sigma_{y}^{2}+\mu_{y}^{2}\right)$

The Log-Normal distribution has the probability density function:

$$
f\left(x, \mu_{y}, \sigma_{y}\right)=\frac{1}{x \sigma_{y} \sqrt{2 \pi}} \exp \left[-\frac{\left(\ln (x)-\mu_{y}\right)^{2}}{2 \sigma_{y}^{2}}\right]
$$

The headcount poverty or the cumulative distribution function is given by:

$$
H=\frac{1}{2}+\frac{1}{2} \operatorname{erf}\left(\frac{\ln \left(z-\mu_{y}\right)}{\sigma_{y} \sqrt{2}}\right)
$$




\section{Appendix 3 Estimation of elasticity and semi- elasticity with Stata}

Two Stata modules (efgtgro and efgtine) are already programmed and can be downloaded from internet. 14 These two modules mainly enable the estimation of elasticity and semi-elasticity of poverty with respect to growth or inequality, and this, with the different approaches presented in this paper. For instance, to produce the results of Figure 11, from the Stata command window, we have to type the command line: db efgtgro, and then, we have to indicate the variables of interest and to select the options, as shown in Figure 13. After clicking on the

Figure 13: The dialog box to estimate the elasticity of poverty with respect to growth

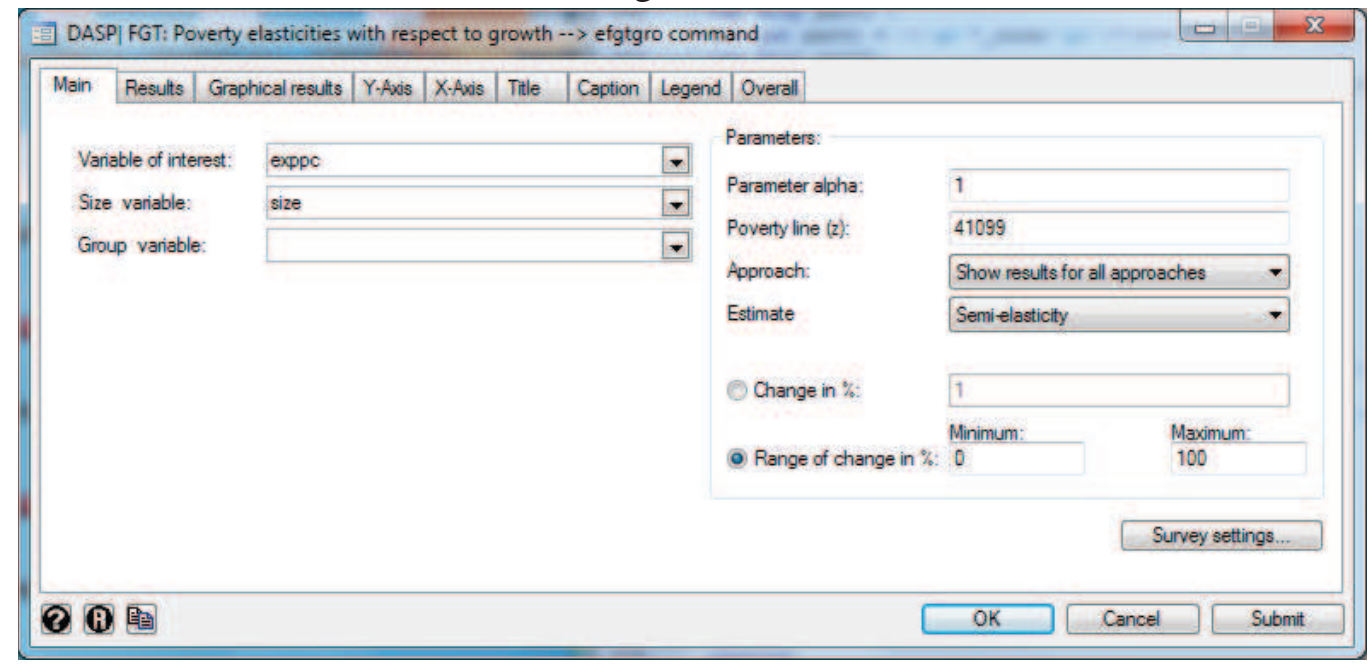

button OK, the graph of Figure 11 is automatically generated (see the Figure 14).

\footnotetext{
${ }^{14}$ To install these two Stata modules, from the Stata command window, type the command: net from http://dasp.ecn.ulaval.ca/efgtgi. Further, these two modules will be integrated of DASP 2.2 (see also the web page in DASP at:http://dasp.ecn.ulaval.ca).
} 
Figure 14: The reproduction of results of Figure 11

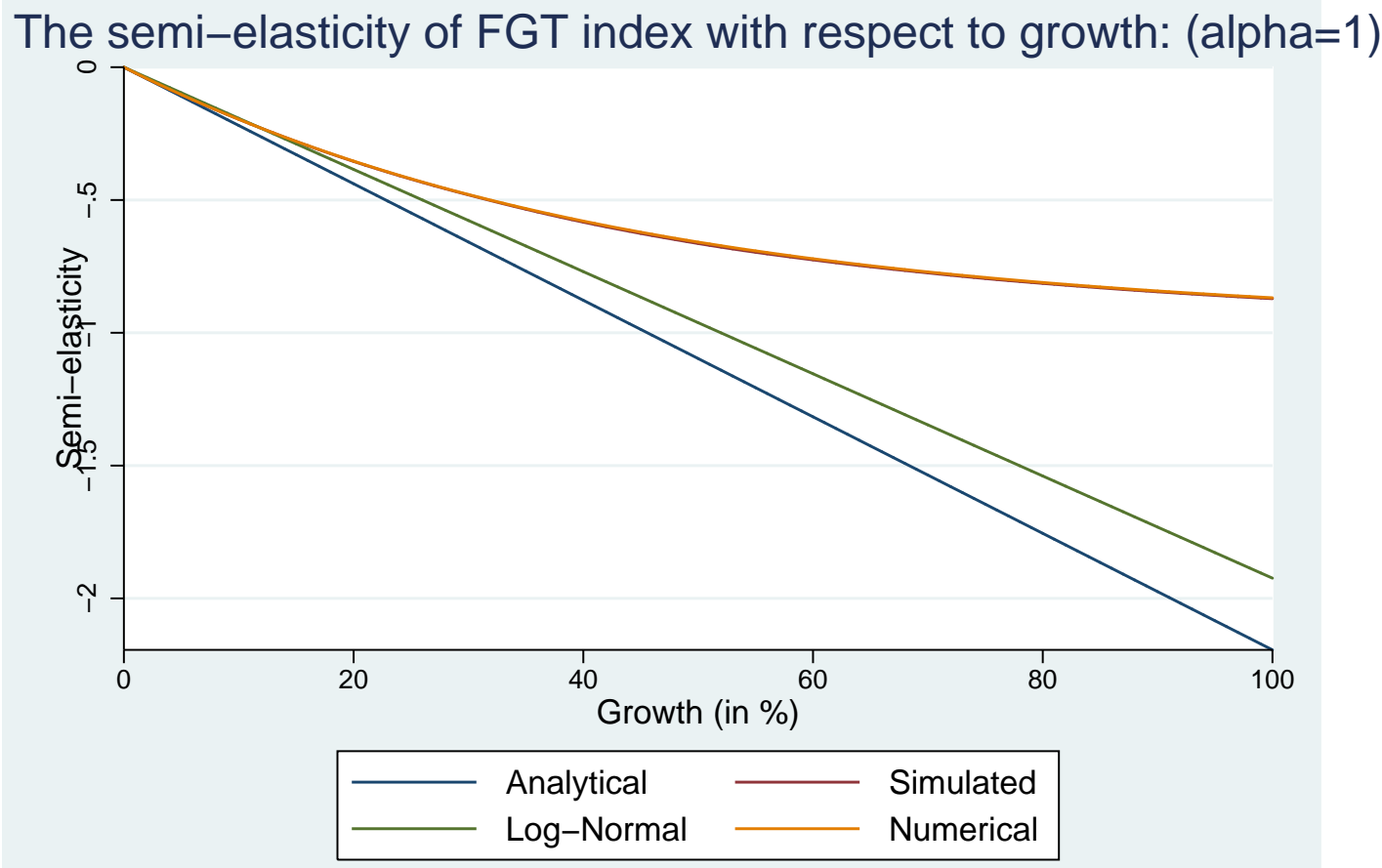

\footnotetext{
${ }^{15}$ Note that, these two modules contain many other options. The user can consult the help for more details on how to use these Stata modules.
} 